\title{
A Semi-Automatic Approach in GIS for 3D Modeling and Visualization of Utility Networks: Application for Sewer \& Stormwater networks
}

\author{
Fadi Chaaban ${ }^{1 *}$, Hanan Darwishe ${ }^{2}$, and Jamal El Khattabi ${ }^{3}$ \\ ${ }^{1}$ Dep. of Topographic Eng., Faculty of Civil Engineering, Tishreen University, Lattakia, Syria. \\ ${ }^{2}$ Dep. of Topographical Eng., Faculty of Civil Engineering, AlBaath University, Homs, Syria. \\ ${ }^{3}$ Civil Engineering and Geo-Environment Laboratory (LGCgE), Lille University, France.
}

\begin{abstract}
This paper presents a semi-automatic methodology proposed for 3D modeling of utility networks in GIS environment. The ModelBuilder in ArcGIS (ESRI) software is used for implementing this methodology, by developing two tools to automate the construction processes of 3D networks. The first presents a tool to create a 3D Manhole layer from points layer, and the second is a tool to create a 3D pipe layer. For both tools, a work algorithm has been built, in addition to designing user interfaces elements. These tools are stored in a Toolbox called "3D Manhole \& Pipe.tbx". The two previous tools were tested and applied to spatial data for a proposed residential area. The final 3D model of the residential area includes the sewage and stormwater networks, as well as other spatial data such as buildings, parks, roads, etc. This model is able to spot the intersection points in the network, visually or using the $3 \mathrm{D}$ analysis available in the software, allowing us to identify problems to be processed and resolved before starting a project, leading consequently to time and cost savings, effort and money. The proposed methodology is an easy and an effective way to build 3D network models (sewer, water..etc), and the developed tools allow the implementation of a set of necessary processes needed to build 3D networks.
\end{abstract}

Keywords: GIS, 3D Utility Networks, 3D Modeling, ArcGIS, ModelBuilder.

\footnotetext{
${ }^{*}$ Corresponding author : fadi.chaaban@tishreen.edu.sy
} 


\section{Introduction}

In general, several researches have studied 3D modeling of cities in the GIS environment $[1,2]$. The development of 3D urban models, both above and underground, allows for new approaches to urban planning and management $[3,4]$. These approaches serve in emergencies, disaster planning, urbanization verification, and infrastructure networks.

Geographic Information Systems (GIS) have a great ability to represent, display, query and manage spatial data (2D or $3 \mathrm{D})$. It also has the ability to manage utility networks such as sewerage, Stormwater networks, water distribution, telephone networks... etc. The representation of 2D networks is related to various obstacles, such as overlapping lines that represent different networks, and various vertical elements (manholes). Moreover, 3D analysis is not possible using 2D geometry. Therefore, attention to the 3D representation of these infrastructure networks has increased rapidly in recent times [4].

Three-dimensional modeling of utility networks is an important mean of networks design, implementation, management and maintenance. During the modeling process, a wide range of processes and procedures was faced to reach the final model. This research proposes a semi-automatic methodology for 3D modeling of infrastructure networks in GIS environment, by developing two tools to automate the construction processes of $3 \mathrm{D}$ networks.

The provided methodology in this research contributes to building network models in an easy and an efficient way that saves time and effort, and ensures a correct way to build the spatial and topological relationships between different components of the networks. This methodology includes the following objectives: (1) Building a Geodatabase for infrastructure networks of a small residential area, (2) Development of two tools to automate the construction processes of $3 \mathrm{D}$ networks in the model builder environment (ArcGIS platform), (3) Export the model and all its elements to a WebScene interface to make it easily available to users, and to exploit the data associated with it.

\section{Material and Methods}

\subsection{Study area}

The study area is located in east of Tartous city centre (Syria) (about $3 \mathrm{~km}$ ), between longitude $35.9236^{\circ} \mathrm{E}$ to $35.93029^{\circ} \mathrm{E}$, and latitude $34.8976{ }^{\circ} \mathrm{N}, 34.8901{ }^{\circ} \mathrm{N}$ (Fig. 1). It covers an approximately area of 16 hectares. It is a proposed residential area by [1]. The residential area consists of 54 residential blocks. It includes also commercial area (with a floor area of $3600 \mathrm{~m}^{2}$, gardens, playgrounds and water bodies. Geodatabase was built by dividing the space into a group of layers: buildings, road network, malls, schools, kindergartens, parks, playgrounds, water bodies, etc. based on WGS 84 / UTM zone $36 \mathrm{~N}$ projected coordinate system.

\subsection{Work platform and software}

Essentially, this research is based on the ArcGIS platform developed by ESRI [5], ArcGIS is a platform to create, manage, share, and analyse spatial data [6]. It allows representing spatial data in 2D and 3D. It has been used in many of global scientific researches such as $[7,8]$. 


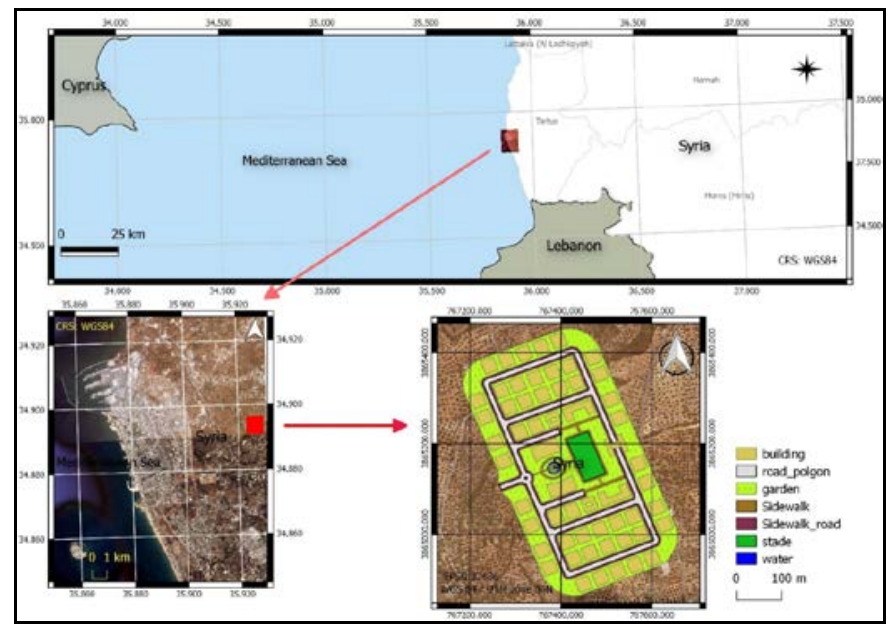

Fig. 1. Study area

\subsection{ModelBuilder and geoprocessing in ArcGIS}

\subsubsection{Geoprocessing}

Geoprocessing generally depends on the systematic implementation of process Series that are applied to spatial data to create new layers (new derived spatial data). ArcGIS consists of two environments for completing geoprocessing, namely ArcToolbox and ModelBuilder.

\subsubsection{ModelBuilder}

ModelBuilder is a visual programming language to create, modify, and manage models within ArcGIS. It is used to automate workflow using the geoprocessing tools available within ArcGIS (ArcToolbox) [9]. There are two basic modes for creating ModelBuilder. The first mode allows exploratory work to be performed. The other one is an application where generic tools can be built, reused and shared. In 2012, [7] designed two general models using ModelBuilder that can be used for geoprocessing linear features (calculate changes between two linear elements). Many researchers have also used ModelBuilder in global scientific research such as: [8] to calculate the change in night lights in China, and [10] who implemented a tool for facilitating the process of urban regional risk assessment.

\subsection{Preparing spatial data for $3 \mathrm{~d}$ modeling}

\subsubsection{Creating layers}

The necessary feature classes of modeling (the sewer and Stormwater networks layers) were created within ArcCatalog, where each feature class was grouped into its own feature dataset (Fig. 2). These layers are: network pipes (line feature class) and network manholes (point feature class). Fig.2 shows the attribute tables of each feature class. line feature class includes the following fields: ID, Name, Diameter $(\mathrm{mm})$, BtmPipeStart (m), MIdPipeStart (m), BtmPipeEnd(m), slope_Thousand, Depth_Start (m), Depth_End (m), DEM_Design (m), Shape_Length (m). Point feature class includes 
the following fields: ID, Name, elvation_designe (m), input1 (m), input2 (m), output (m), Depth (m).

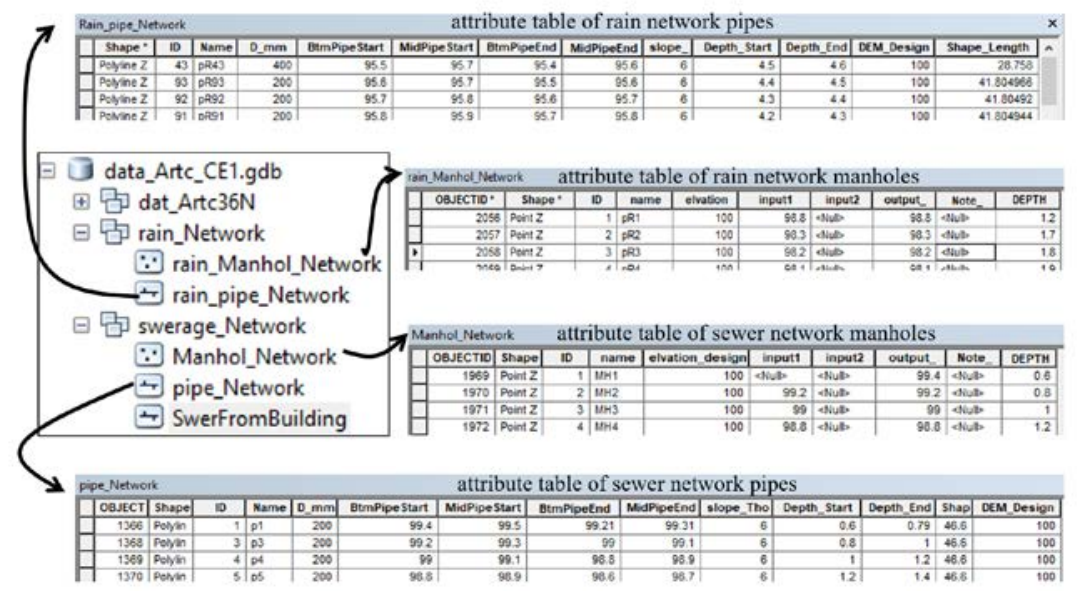

Fig. 2. Spatial database and attribute tables of networks pipes and manholes

To verify the data quality, a new topology was created for both networks. some topology rules of ArcGIS were used such as: Must Not Overlap, Must Not Intersect, Must Not Have Dangles, Must Not Self-Overlap, Must Not Self-Intersect, Must Be Covered By Endpoint Of, Must Be Single Part, Endpoint Must Be Covered By. The errors were corrected or excepted. Fig. 3 shows the 2D corrected spatial data (pipes and manholes) for both networks (sewer and rain) after applying the symbology.

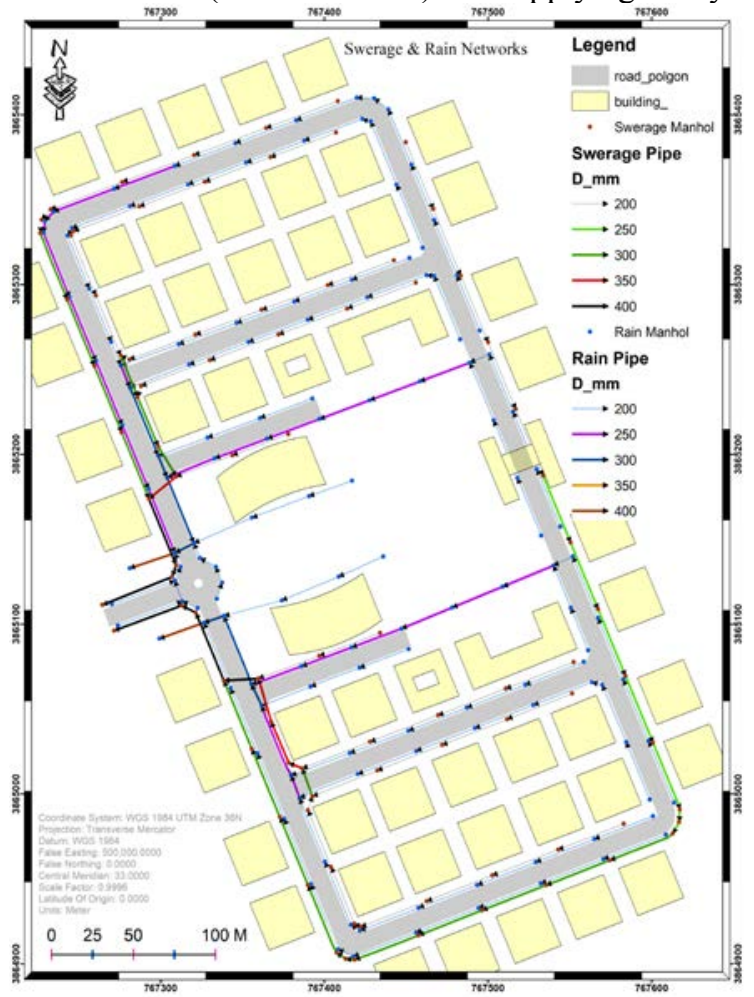

Fig. 3. 2D spatial data (networks pipes and manholes) 


\section{Results and discussion}

\subsection{Automating the construction processes of 3D networks}

In this stage, two tools were developed (in ModelBuilder) to automate the construction processes of 3D networks from $2 \mathrm{D}$ layers. The first presents a tool to create a 3D Manhole layer from points, and the second is a tool to create a 3D pipe layer from lines. For both tools, a work algorithm has been built (Fig.4 a, b), in addition to designing user interfaces elements (Fig.4 c, d). These tools are stored in a Toolbox called "3D Manhole \& Pipe.tbx".
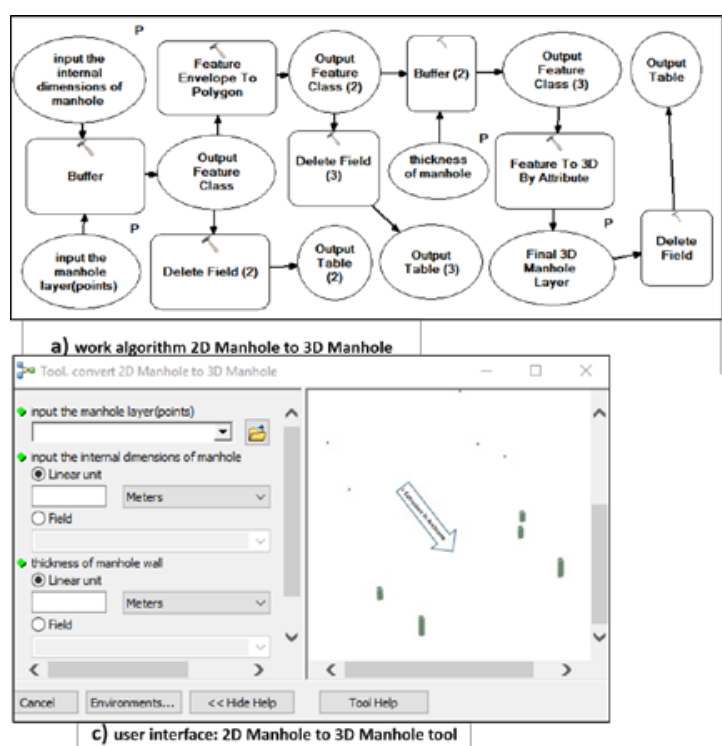

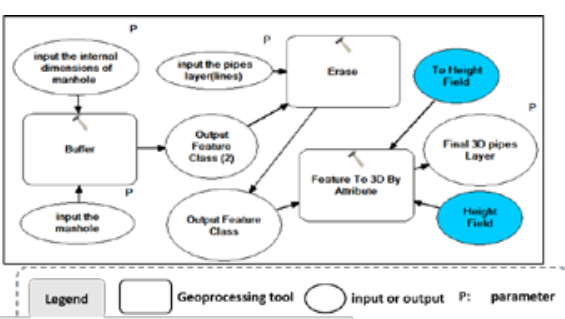

b) work algorithm 20 pipes to 30 pipes

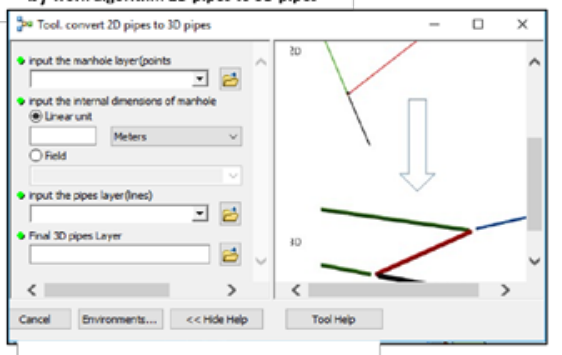

d) user interface: $2 \mathrm{D}$ pipes to $3 \mathrm{D}$ pipes tool

Fig. 4. Work algorithm and user interfaces

Fig.4 (a) presents a work algorithm for converting 2D manhole to 3D. It consists of the following tools: Buffer, Feature Envelope to Polygon, Delete Field, Feature To 3D By Attribute and the Outputs of each tool. Fig.4 (b) presents a work algorithm for creating a 3D pipe layer from lines. It consists of the following tools: Buffer, Erase, Delete Field, Feature To 3D By Attribute and the Outputs of each tool.

\subsection{Test and apply the developed tools}

The two previous developed tools were tested and applied to the spatial data presented previously (in 2.4 paragraph), which contain the required and necessary fields. Firstly, the 2D Manhole layers were converted to 3D Manhole layers, then the 2D pipes layers were converted to 3D pipes layers. In the end, three-dimensional layers $(\mathrm{x}, \mathrm{y}, \mathrm{z})$ were obtained where the cartographic representation and visualization are possible in an environment that reads this type of 3D data (such as ArcScene). Fig. 5 represents the final 3D model of the residential area including the sewer and Stormwater networks (Manholes \& Pipes), as well as other spatial data such as buildings, gardens, roads, etc. Through this model, This model is able to spot the intersection points in the network, visually or using the $3 \mathrm{D}$ analysis available in the software, allowing us to 
identify problems to be processed and resolved before starting a project, leading consequently to time and cost savings, effort and money.

The final stage of the modeling process is to share the project with other users (using ArcGIS online) by exporting the 3D model with all its elements to a WebScene interface to make it readily available to users, and to exploit the data associated with it. This interface allows to search for any feature, as well as to display the attribute data associated with any selected feature. A set of selected scenes and created bookmarks can also been exported, that can be viewed as a video within a web page.

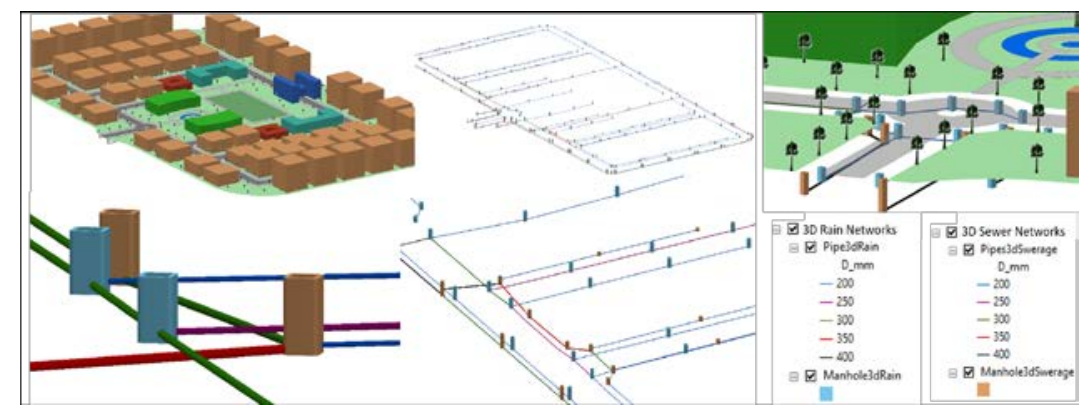

Fig. 5. Final 3D model of the residential area including the sewer and Stormwater networks

\section{Conclusion}

In this research, a semi-automatic methodology was proposed for 3D modeling of infrastructure networks in GIS environment, by developing two tools in order to automate the construction processes of $3 \mathrm{D}$ networks. A range of results was obtained: (i) The use of GIS reduces the effort to prepare spatial and attribute data for digital models, and is widely used as a tool to assist analysis, planning, management and decision-making. (ii)The ModelBuilder is an easy-to-use application to accomplish multiple and complex work tasks. (iii) The methodology presented is flexible and adaptable according to the status and type of Utility Networks. (iv)The $3 \mathrm{~d}$ model can be shared with other users as WebScene which can be readily and exploitable. 


\section{References}

1. H. Darwishe, F. Chaaban, Procedural 3D modeling of cities in Geographic Information Systems environment using ESRI CityEngine (published in Arabic). Albaath univ. magazine. 39,11(2017)

2. K. N. Edvardsson, 3D GIS modelling using ESRI's CityEngine: a case study from the University Jaume I in Castellon de la Plana Spain (Doctoral dissertation), (2013).

3. J. Benner, A. Geiger, K. Leinemann, F. et al., Flexible Generation of Semantic 3D Building Models, Work. Next Gener. 3D City Model, 49, pp. 17-22.(2005)

4. T. Becker, C. Nagel, And T. H. Kolbe. Semantic 3D modeling of multi-utility networks in cities for analysis and $3 D$ visualization. in Lecture Notes in Geoinformation and Cartography, pp. 41-62 (2013)

5. ESRI, "Environmental Systems Research Institute,". [Online]. Available: https://www.esri.com. [Accessed: 01-Apr-2019].

6. ArcGIS for Developers. [Online]. Available: https://developers.arcgis.com. [Accessed: 01-Apr-2019].

7. F. Chaaban, H. Darwishe, Y. Battiau-Queney, B. Louche, E. Masson, J. El Khattabi, E. Carlier. Using ArcGIS Modelbuilder and Aerial Photographs to Measure Coastline Retreat and Advance: North of France," J. Coast.Res.,285, pp.1567-1579(2012)

8. S. Scheider And A. Ballatore. Semantic typing of linked geoprocessing workflows, International Journal of Digital Earth, pp. 1-9.(2017)

9. D. W. Allen.Getting to Know ArcGIS ModelBuilder(Redlands,CA:Esri Press,2011)

10. M. Zhao And X. Liu. Regional risk assessment for urban major hazards based on GIS geoprocessing to improve public safety, Saf. Sci. 87, pp. 18-24 (2016) 